\title{
EchoGéo
}

$8 \mid 2009$

Moyen-Orient : conflits et mobilités dans un espace mondialisé

\section{Le Sénégal Oriental à l'aube du développement minier}

Les collectivités locales des jeux du pouvoir aux enjeux du territoire

Isabelle Greig

\section{OpenEdition}

Journals

Édition électronique

URL : https://journals.openedition.org/echogeo/10918

DOI : 10.4000/echogeo.10918

ISSN : 1963-1197

Éditeur

Pôle de recherche pour l'organisation et la diffusion de l'information géographique (CNRS UMR 8586)

\section{Référence électronique}

Isabelle Greig, « Le Sénégal Oriental à l'aube du développement minier », EchoGéo [En ligne], 8| 2009

mis en ligne le 25 février 2009, consulté le 31 juillet 2021. URL : http://journals.openedition.org/ echogeo/10918; DOI : https://doi.org/10.4000/echogeo.10918

Ce document a été généré automatiquement le 31 juillet 2021.

EchoGéo est mis à disposition selon les termes de la licence Creative Commons Attribution - Pas d'Utilisation Commerciale - Pas de Modification 4.0 International (CC BY-NC-ND) 


\title{
Le Sénégal Oriental à l'aube du développement minier
}

\author{
Les collectivités locales des jeux du pouvoir aux enjeux du territoire
}

\author{
Isabelle Greig
}

1 A la faveur d'une remontée du cours des matières premières au début des années 2000, le Sénégal relance un vieux projet: l'exploitation des richesses minières du Sénégal Oriental (or, fer, marbre). Le développement d'activités extractives ouvrirait des perspectives en termes d'industrialisation et d'aménagement du territoire à travers la promotion d'une région périphérique et enclavée. Ce travail qui se place à l'échelle de la région de Tambacounda, se propose d'analyser les perspectives d'évolution d'un territoire sous l'effet conjugué de trois dynamiques: le développement des activités extractives, la mise en œuvre d'une politique d'aménagement du territoire et les balbutiements de la décentralisation.

2 La décentralisation est devenue depuis les années 1990 un thème majeur du discours sur le développement, solution démocratique aux défaillances des Etats et garantie d'un développement local concerté (Gentil et Husson, 1996 ; Totte et al., 2003 ; Magrin, 2006). Le Sénégal est l'un des pays africains où ce processus est le plus avancé. Si les activités minières ne sont pas du ressort des collectivités locales, plusieurs compétences transférées entrent en ligne de compte dans ce domaine : environnement, planification, aménagement du territoire, formation, développement économique.

3 Il s'agira de mettre en évidence un jeu d'intérêts contradictoires et d'échelles spatiales imbriquées qui met en tension les différents acteurs du développement minier, de l'Etat aux collectivités locales et des sociétés minières à la société civile. Lequel de ces pôles influence le plus les dynamiques territoriales? Il s'agira ici de déterminer le rôle de chacun dans un jeu dissymétrique à la fois en termes de pouvoirs et en termes d'échelles spatiales de référence. Nous organiserons notre réflexion autour du double défi auquel doivent faire face les collectivités locales: s'affirmer dans des jeux de pouvoir pour réinventer un (des) territoire(s).

4 Cet article présente les conclusions d'un mémoire de master 1 de géographie soutenu en 2006 à l'issue d'enquêtes menées dans le cadre d'un stage de trois mois au CIRAD et à 
l'Agence Régionale de Développement de Tambacounda (ARD) ${ }^{1}$. L'angle d'approche privilégiant les collectivités locales rejoignait l'intérêt commun de l'ARD, du CIRAD et du laboratoire GIRARDEL de l'Université de Saint-Louis (Sénégal) ${ }^{2}$ pour les questions liées à la décentralisation. A sa dimension prospective fait écho le retournement de conjoncture actuel: si l'or semble rester une valeur refuge en ces temps de crise économique mondiale, il y a fort à parier que le projet d'exploitation du fer de la Falémé sera à nouveau reporté sine die.

\section{S'affirmer dans des jeux de pouvoir...}

\section{Des firmes transnationales inscrites dans des jeux d'acteurs mondialisés}

5 L'économie minière de la région doit reposer sur les mines d'or et de fer ainsi que sur l'exploitation des carrières de marbre (voir carte 1). L'exploitation de l'or fait intervenir cinq compagnies minières transnationales. Une seule s'apprête à entrer en exploitation: la société australienne Mineral Deposits Limited (MDL), implantée à Sabodala. Les ressources sont estimées à 30 tonnes (soit environ quatre tonnes par an pendant sept ans ${ }^{3}$ ). Trois sociétés canadiennes et une société sud-africaine mènent des activités d'exploration. L'exploitation du fer fait l'objet de négociations entre la Société des Mines de fer du Sénégal Oriental (MIFERSO), détenue par l'Etat sénégalais, et la première compagnie sidérurgique mondiale, Mittal Steel. L'exploitation pourrait commencer à l'horizon 2011, à un rythme de 25 millions de tonnes par an4

Carte 1 - Activités et potentiels miniers (or, fer, marbre)

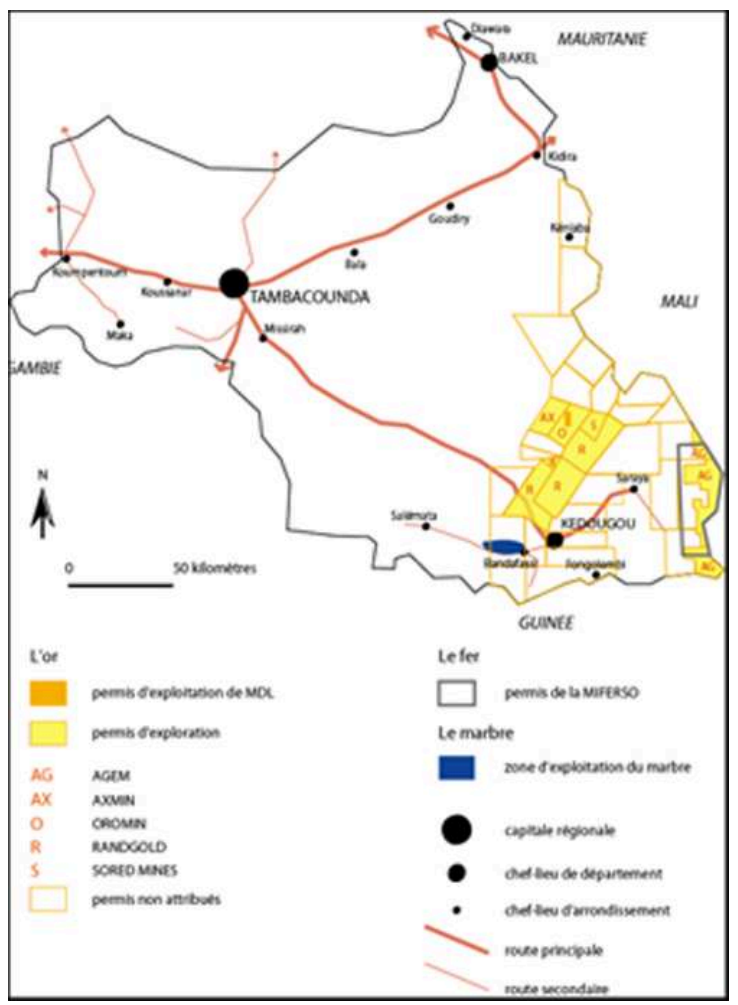

Carte réalisée par Isabelle Greig, d'après des documents fournis par la Direction des Mines et de la Géologie, mai 2006. 
6 L'exigence de profit des grandes industries minières combinées aux conditions spécifiques des pays du Sud (législation plus faible notamment en matière de protection de l'environnement, multiplicité des foyers de conflit, pauvreté, SIDA...) fait peser sur une probable future région minière comme le Sénégal Oriental des menaces d'ordre à la fois environnemental, social et sanitaire. Or, les grandes sociétés minières doivent aujourd'hui compter avec de puissants contre-pouvoirs. A titre d'exemple, l'ONG Oxfam America, implantée au Sénégal, a fait partie des leaders de la campagne No Dirty Gold lancée en 2004. Les dénonciations portent également sur le manque de transparence qui règne dans le monde des industries extractives, elles font l'objet d'un rapport en cours du Forum Civil, section sénégalaise de Transparency International. Cette multiplication des témoignages de vigilance de la société civile incite les opérateurs miniers à intégrer des mesures sociales et environnementales dans leurs activités. Soucieuses de leur image, celles-ci rentrent volontiers dans le moule du " développement durable».

\section{Un Etat omniprésent mais vulnérable}

7 Dans le cadre d'une politique volontariste pour hisser le Sénégal dans le groupe des pays émergents, le gouvernement de l'alternance ${ }^{5}$ a tenté de saisir l'opportunité offerte par un contexte international favorable pour attirer les investisseurs. Le Sénégal s'est ainsi doté depuis 2003 d'un nouveau code minier d'orientation libérale, revendiquant un alignement sur les politiques minières des pays de la sous-région. Plusieurs responsables de sociétés minières se plaignent pourtant de cette législation encore jugée inadaptée. La part que se réserve l'Etat dans le capital des sociétés minières (10\% d'actions gratuites et jusqu'à $20 \%$ de participation onéreuse) est considéré comme trop importante. En outre, l'Etat n'a pas procédé à la délimitation des couloirs d'orpaillage, comme le souhaitaient vivement les sociétés industrielles, qui entrent régulièrement en conflit avec les orpailleurs artisanaux présents dans leurs périmètres.

8 Face aux pressions des sociétés minières, l'Etat sénégalais manque surtout cruellement de capacités techniques qui lui permettraient de négocier à armes égales. La Direction des Mines a récemment mis en place un programme d'appui au secteur minier qui vise à la réalisation d'un cadastre minier. Or, l'absence d'un tel document à l'heure où se développent plusieurs projets miniers paraît alarmante. L'Etat n'a de plus pas les capacités financières suffisantes pour mettre en place les organismes de suivi et de contrôle nécessaires en matière géologique, financière et environnementale. En l'absence de formation spécifique dans le domaine minier, le Lycée Technique Industriel et Minier de Kédougou assure plutôt une mission d'appui à l'artisanat local. Par ailleurs, le Sénégal ne disposant pas de laboratoire d'analyse performant, la plupart des sociétés minières de la région envoient leurs échantillons au Mali ou dans leurs pays d'origine respectifs.

9 Incapable de faire face aux pressions comme aux besoins des sociétés minières, l'Etat confisque de surcroit la négociation avec les opérateurs à son profit. Le code minier comme les conventions signées avec les sociétés minières ont été élaborés sans la moindre consultation des collectivités locales concernées. Tout au plus sont-elles conviées à faire de la figuration lors de la signature des conventions minières. La décentralisation sénégalaise se heurte à l'inertie d'un système auparavant hypercentralisé. Régions et communautés rurales ont acquis en 1996 le statut de collectivités 
décentralisées, plusieurs compétences dont la gestion des ressources naturelles, la gestion domaniale, la planification, l'aménagement du territoire et l'action sociale leur sont transférées. Un transfert concomitant de ressources est prévu pour permettre aux collectivités d'assumer ces compétences. Mais la prise en charge des compétences transférées reste globalement faible car elle se heurte à trois obstacles principaux. Tout d'abord, les textes manquent de clarté dans la répartition des compétences transférées entre les collectivités locales. De plus, le pouvoir de l'Etat reste fort, les représentants locaux de l'Etat (gouverneur, préfet, sous-préfet) exerçant un contrôle sur les décisions prises par les collectivités locales. Enfin, les transferts de l'Etat restent insuffisants (environ $2 \%$ du budget de l'Etat).

Dès lors, l'Etat sénégalais se trouve paradoxalement à la fois omniprésent et dans l'incapacité technique et financière de négocier sur un pied d'égalité avec les opérateurs miniers. Le problème prend une autre dimension lorsqu'on sait que les hautes sphères de l'Etat ont entretenu depuis l'Indépendance des amitiés plus ou moins opaques dans le secteur des mines.

\section{Région et communautés rurales : les collectivités locales isolées}

11 Les nouvelles lois de décentralisation érigent la région en collectivité locale et lui attribuent un rôle d'intermédiaire entre l'Etat central et les collectivités locales de base (Magrin, 2006; Piveteau, 2005). Cependant, la région ne dispose pas de ressources fiscales propres, et ses interventions sont souvent perçues par les communes et communautés rurales comme des velléités hégémonistes. La région assume donc difficilement son rôle d'intermédiaire, dont le terrain privilégié est la planification, à l'articulation du Plan élaboré au niveau national et des outils de planification des collectivités locales de base. La région de Tambacounda est l'une des rares à ne pas avoir actualisé son Schéma Régional d'Aménagement du Territoire. Il faut ajouter à cela des difficultés spécifiques à cette région, qui souffre principalement de son isolement politique. Le Président du Conseil Régional a été l'une des figures importantes du régime socialiste de Senghor, sous lequel il a occupé plusieurs postes ministériels avant de devenir Président de l'Assemblée Nationale. Depuis la victoire du Parti Démocratique Sénégalais d'Abdoulaye Wade, il se retrouve dans l'opposition, de même que les communes de Tambacounda, Bakel et Kédougou, face à un gouvernement et à une majorité de communautés rurales qui sont passés dans le camp de «l'alternance ». Il se pourrait donc que la région soit en train de payer ses choix politiques.

12 A l'échelle locale, les communautés rurales ${ }^{6}$ souffrent quant à elles d'un isolement entre l'autorité coutumière des chefs de villages et l'autorité administrative locale incarnée par les sous-préfets, qui accaparent le dialogue avec les opérateurs miniers. Le chef de village est nommé par le préfet, cependant un décret précise qu'il est placé sous la double autorité du président de la communauté rurale et du sous-préfet. Les interrelations entre les trois pouvoirs sont donc complexes, et ce sont les conditions locales qui déterminent dans une large mesure leur fonctionnement. Or, les communautés rurales souffrent de sérieux handicaps dans l'exercice du pouvoir. Le taux de recouvrement de la taxe rurale est proche de zéro. Le fonctionnement de la communauté rurale s'appuie donc sur le fonds de dotation de l'Etat ainsi que sur un fonds de concours censé pallier le faible taux de recouvrement de la taxe. A cela s'ajoute le manque de compétences de la plupart des conseillers ruraux, en majorité 
analphabètes et sans expérience en matière de négociation avec des opérateurs. Ces difficultés sont plus marquées dans les communautés rurales du Sud-est que dans la basse vallée du Sénégal ou dans les zones urbaines de l'Ouest.

C'est dans ce contexte qu'il faut replacer les relations entre les communautés rurales et les sociétés minières qui interviennent sur leur territoire. L'interlocuteur privilégié voire unique des compagnies minières reste la Direction des Mines. Lorsqu'elles recherchent un interlocuteur localement, les compagnies s'adressent plus volontiers aux chefs de villages et à l'autorité administrative qu'au conseil rural. Le personnageclé du dialogue à l'échelle locale reste le sous-préfet, qui garde la haute main sur le recrutement des ouvriers. Il est difficile de faire la part de la méconnaissance des structures de la décentralisation et de celle d'une volonté délibérée de contourner le seul pouvoir dont la légitimité est celle des urnes. S'adresser aux chefs de village s'apparente à une posture à la fois démagogique et peu risquée, car ils ont peu de pouvoir réel et se satisfont de quelques «cadeaux", selon l'expression du chef du village de Sabodala.

C'est autour de Sabodala, dans la communauté rurale de Khossanto, que la procédure de concertation est la plus aboutie, les enjeux les plus forts, et les jeux de pouvoir les plus perceptibles. Un cadre de concertation a été constitué, présidé par le sous-préfet, qui fait intervenir des représentants de MDL et OROMIN, le président de la communauté rurale, un représentant de la Direction des Mines et les chefs des villages environnants. La société OROMIN ne s'est fait représenter qu'une fois et reste mal connue des chefs de village comme de la communauté rurale. Le chef de projet de MDL, de loin le plus engagé dans le dialogue à l'échelle locale, affirme clairement reconnaître la légitimité de la communauté rurale de Khossanto. Cependant celle-ci se voit fragilisée par des dissensions internes, le Président de la communauté rurale ayant fait l'objet d'une procédure de destitution en 2006 : la confusion entre la personnalité du président et l'institution elle-même en paralyse le fonctionnement.

\section{...pour réinventer un (des) territoire(s)}

\section{S'intégrer au territoire national}

15 Le Sénégal Oriental fait figure de marge enclavée au sein d'un espace national dominé par Dakar et la frange littorale. Sa participation au produit intérieur brut représente moins de $3 \%$, ce qui traduit sa marginalité économique. La densité de la population à l'échelle de la région se situe autour de 8 habitants au $\mathrm{km}^{2}$. La ville principale, Tambacounda, reste loin derrière les grandes villes de l'ouest du pays, avec seulement 67000 habitants en 2002.

Cette situation marginale est rendue plus sensible encore par l'enclavement qui caractérise la région, lié non seulement à la distance, mais surtout à la faiblesse qualitative et quantitative des infrastructures de transport et de communication, qui entravent les circulations internes et les relations avec la région-capitale et les pays voisins (Debrie, Steck, 2001 ; Magrin, Ninot, 2005). Dans le domaine des équipements, le Sénégal Oriental fait en effet figure de "région oubliée ", selon une expression fréquemment reprise par les acteurs locaux. De fait, Tambacounda et Bakel n'ont été rattachées au réseau routier bitumé que dans le courant des années 1980, Kédougou seulement en 1996. Le transport ferroviaire sur la ligne Dakar-Bamako, réservé au fret, 
pâtit d'infrastructures vétustes et reste peu important. Il n'existe pas de ligne aérienne régulière reliant Tambacounda à Dakar. La région accuse également du retard sur le plan des télécommunications : le développement de la téléphonie rurale est en cours avec le raccordement de tous les chefs-lieux d'arrondissement.

Carte 2 - Enjeux d'aménagement du territoire au Sénégal Oriental

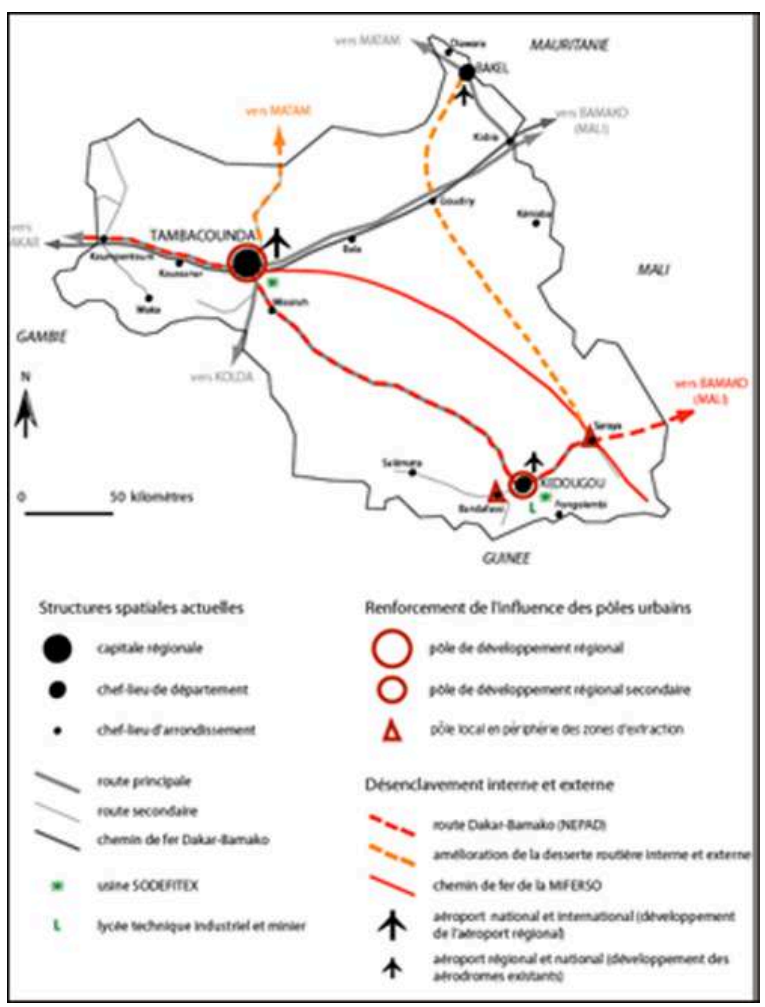

Carte réalisée par Isabelle Greig, mai 2006

En termes d'aménagement du territoire, l'émergence des activités minières constitue une opportunité nouvelle (voir carte 2). L'acheminement de matériaux lourds comme le fer et le marbre suppose un meilleur raccordement au centre-ouest du pays. L'évacuation du minerai de fer nécessite la construction de 300 kilomètres de voie ferrée entre la mine de Kodi Kourou et Tambacounda et la rénovation de la voie ferrée existante entre Tambacounda et la région-capitale. La création d'une usine de première transformation du fer sur place est en projet. Une première transformation du marbre pourrait également avoir lieu à Bandafassi, mais l'essentiel du traitement industriel devrait s'opérer dans la grande banlieue de Dakar. Les retombées positives concerneraient sans doute au premier chef Dakar et ses environs immédiats à travers le développement du port minéralier en eau profonde de Bargny, qui fait partie des grands travaux projetés par le gouvernement ${ }^{7}$. Néanmoins, le Sénégal Oriental pourrait voir s'étoffer ses infrastructures de transport et de communication ainsi que le réseau des villes régionales par la croissance de petits pôles situés dans la périphérie des zones d'extraction tels que Saraya et surtout par le renforcement des villes principales. On peut ainsi envisager un accroissement et une complexification des fonctions de Tambacounda, par la création d'unités industrielles de première transformation des minerais mais aussi des produits agricoles locaux, ainsi que par l'expansion du secteur commercial et bancaire. 


\section{Reconsidérer la « vocation » régionale}

18

Le Sénégal Oriental semble avoir conservé jusqu'à aujourd'hui au sein du territoire sénégalais un statut d'espace de réserve aux potentialités encore inexploitées. L'apparent paradoxe d'une région qui serait à la fois la plus pauvre par ses conditions de vie et la plus riche par ses potentialités revient comme un leitmotiv dans la bouche des différents acteurs. La « vocation » régionale telle qu'énoncée jusqu'à l'apparition des projets miniers serait d'être un conservatoire de la nature sauvage et d'un mode de vie traditionnel. L'économie régionale repose largement sur le secteur primaire alors que l'industrialisation reste à un stade embryonnaire : elle se limite aux deux usines d'égrenage du coton de la SODEFITEX, à Tambacounda et Kédougou.

Or, lorsque l'exploitation de « réserves » qui relevaient jusque là de la pure spéculation semble se concrétiser, les perspectives de développement liées aux activités minières entrent en tension avec les logiques de protection et de valorisation du patrimoine naturel (voir carte 3). Le Sénégal Oriental se caractérise en effet par l'importance de ses espaces naturels classés, parmi lesquels se distinguent le Parc National du Niokolo Koba et la Zone d'Intérêt Cynégétique de la Falémé. Cette dernière semble la plus menacée car les principaux gisements d'or et de fer s'y concentrent. Les entreprises minières sont astreintes à effectuer à leurs frais une étude d'impact environnemental et social qui doit accompagner toute demande de permis d'exploitation. Cependant, cette procédure a d'ores et déjà été mise à mal par la société MDL, qui a obtenu la signature d'une convention minière avant même la réalisation de l'étude d'impact.

Carte 3 - Activités minières : une menace pour l'environnement naturel et économique?

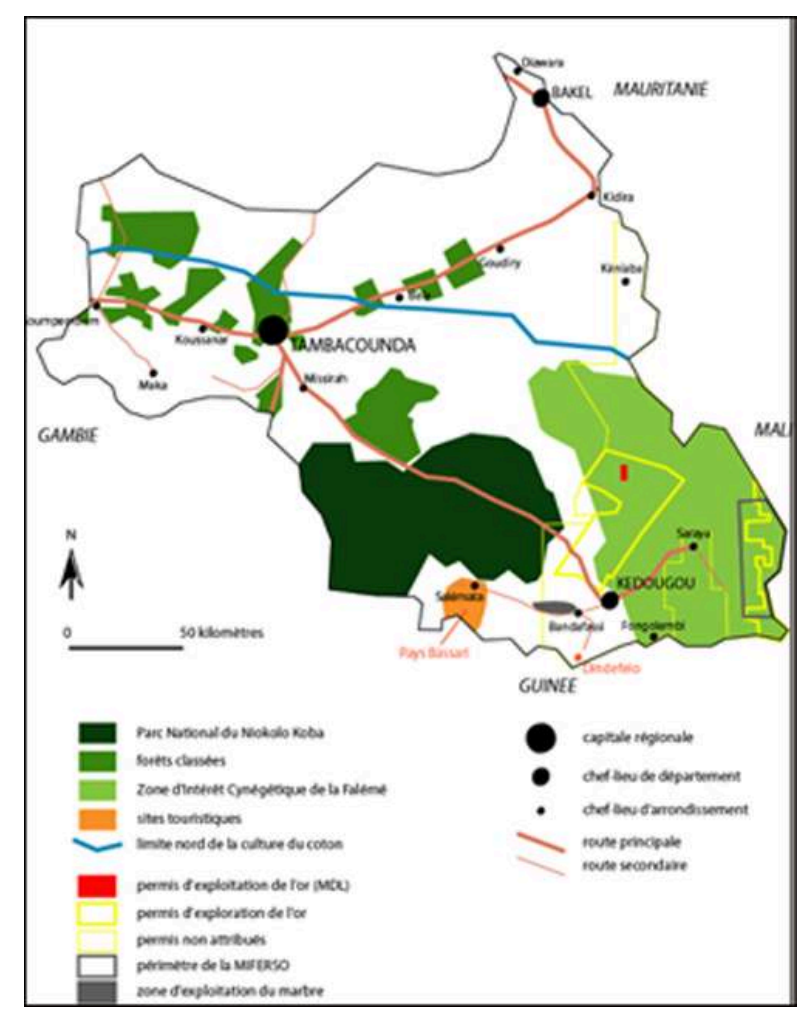

Carte réalisée par Isabelle Greig, mai 2006. 
Ces tensions naissantes et appelées à s'intensifier à mesure de l'avancement des travaux de MDL et des autres sociétés montrent bien que le gouvernement sénégalais, et secondairement les acteurs locaux, se trouvent face à la nécessité d'opérer clairement un choix, et incitent à reconsidérer la notion de "vocation" régionale. Celle-ci a été abondamment utilisée pour justifier a posteriori la "spécialisation » du Sénégal Oriental dans la conservation de grands espaces naturels et suggère implicitement l'absence de réel choix et pour tout dire l'inconsistance des acteurs face à l'évidence du lieu. Elle est finalement symptomatique du peu d'intérêt accordé au Sénégal Oriental dans les plans successifs d'aménagement du territoire. A l'heure où la perspective de l'exploitation minière revalorise la région dans les représentations dominantes, il ne s'agit pas d'envisager la transition d'une « vocation de conservatoire de la nature " vers une "vocation minière " mais bien de substituer à l'évidence supposée de la « vocation » des choix politiques d'aménagement explicités comme tels.

\section{Echapper au piège de l'ultra-local}

21 La gestion de l'extraction minière et de ses impacts sociaux et environnementaux nécessite de les appréhender à différentes échelles. Or, l'attitude des opérateurs miniers comme des ONG et projets de développement présents sur le terrain tend à focaliser plus ou moins délibérément l'attention sur l'échelon ultra-local de quelques villages, en entretenant la confusion entre effets bénéfiques limités dans le temps et dans l'espace et progrès susceptible d'exercer un effet d'entraînement d'échelle régionale à long terme.

22 A titre d'exemple, MDL s'engage à mettre en œuvre un programme social durant la phase de pré-exploitation et pendant toute la durée de l'exploitation. En outre, la société recrute de la main-d'œuvre locale, parfois jusqu'à 450 personnes par jour. Enfin, elle revendique une série d'actions "sociales" : réhabilitation de la piste menant de Khossanto à Sabodala, forage dans le village de Faloumbou, adduction d'eau à Sabodala, réparation et maintenance de moulins à mil, don de gasoil pour faire fonctionner le groupe électrogène de Sabodala, mise à disposition de véhicules de la compagnie pour évacuer des malades, enfin appui financier et matériel aux services de l'Etat et aux chefs de villages.

Cet engagement social des sociétés laisse néanmoins plusieurs problèmes en suspens. Tout d'abord, au-delà du recrutement de main-d'œuvre locale non-qualifiée, les sociétés minières s'apparentent souvent à des enclaves socioculturelles et économiques peu ouvertes sur leur environnement. Les entreprises ont beaucoup recours à la soustraitance, essentiellement étrangère, pour effectuer certaines tâches techniques liées à l'exploration minière. La restauration des campements est assurée par un restaurateur dakarois qui fait tout venir de la capitale en camion frigorifique. Un deuxième problème reste entier : celui de la définition du vocable élastique de "populations locales ». L'expression est d'un usage répandu et commode dans la mesure où elle maintient le flou, et ce doublement. Difficile de préciser si l'échelle du « local » est celle des villages inclus dans le périmètre de la société, de la communauté rurale, de l'arrondissement, ou même du département. De surcroît la notion de " populations " renvoie implicitement à une collectivité humaine monolithique, non hiérarchisée et non politisée, quand la réalité du terrain voit s'affronter des pouvoirs locaux aux intérêts bien compris. De plus la question classique de l'opposition entre autochtones et 
allochtones dans les sociétés villageoises se trouve posée avec d'autant plus d'acuité du fait de l'afflux de migrants lié au développement des activités extractives.

ONG et projets développent également une série d'actions à très grande échelle, victimes d'une sorte de myopie qui ne permet pas de saisir les enjeux de développement à plus petite échelle. On peut citer l'exemple du Groupe d'Action pour le Développement Communautaire. Cette ONG basée à Tambacounda a réalisé un microbarrage et un périmètre maraîcher à l'entrée du village de Khossanto, qui sont entrés en fonctionnement en 2005. Ce projet fait partie des activités génératrices de revenus qui pourraient se développer en profitant de la proximité des industries extractives. D'autres villages du secteur ont d'ailleurs manifesté le souhait de développer des projets identiques. Pourtant le coordonnateur du projet ne semble pas soupçonner l'intérêt du développement de telles activités pour la région et n'a pas cherché à mesurer l'intérêt des sociétés minières du secteur pour le périmètre maraîcher.

L'échelon ultra-local exerce une séduction d'autant plus grande que celui des collectivités locales peine à s'affirmer. L'Agence Régionale de Développement joue dans la limite de ses moyens un rôle d'appui à la décentralisation, en collaboration mais aussi parfois en concurrence avec les autres acteurs du développement présents localement. Les missions confiées à l'ARD sont larges, ce qui nuit à l'efficacité de son action, et son domaine de compétence est mal délimité par rapport à celui des services de l'Etat. Elle manque par ailleurs de moyens financiers et humains. Enfin, sur le plan institutionnel, elle est souvent perçue par les collectivités locales de base comme inféodée au Conseil Régional. Une réforme des ARD devrait accompagner la mise en place du Programme National de Développement Local ${ }^{8}$ actuellement en cours. Cette réforme et plus largement le progrès de la décentralisation semblent être les meilleures réponses à la focalisation sur l'ultra-local.

\section{Conclusion}

L'analyse des rapports entre activités minières émergentes et collectivités locales encore fragiles au Sénégal Oriental croise plusieurs thématiques mises en évidence par la recherche africaniste en sciences humaines. D'abord, le rapport dissymétrique entre une Afrique pourvoyeuse de matières premières et des pays industrialisés perpétuant à travers les firmes multinationales qui en sont issues une domination aux implications sociales et environnementales parfois dramatiques. Ensuite, les limites d'un Etat africain inefficace faute de moyens et de compétences spécialisées, aux institutions calquées sur un modèle bureaucratique jacobin et aux élites souvent corrompues. Enfin, les déceptions de la décentralisation, trop souvent présentée comme une panacée garantissant tout à la fois démocratie à la base et développement local, mais qui se heurte dans la pratique à la faiblesse des moyens humains et financiers et à l'inertie des pouvoirs en place.

Cependant, le Sénégal Oriental, s'il présente une vulnérabilité spécifique aux impacts de l'activité minière du fait notamment de son important patrimoine forestier, bénéficie tout de même d'un contexte national qui reste privilégié en Afrique de l'Ouest : un Etat relativement stable et démocratique et une décentralisation parmi les plus abouties en dépit des difficultés qu'elle rencontre. La médiation des collectivités locales, région et communautés rurales, dans un dialogue faisant intervenir des acteurs multiples, nous paraît aujourd'hui indispensable non seulement en ce qu'elles sont les 
seules à bénéficier de la légitimité démocratique au niveau local, mais surtout en ce qu'elles apparaissent comme des échelons pertinents de la négociation permettant d'éviter une focalisation sur un échelon "ultra-local » instrumentalisé tant par les compagnies minières que par de nombreux programmes de développement. Le niveau régional apparaît en outre comme le plus adapté à la mise en œuvre d'une politique d'aménagement du territoire dont l'intégration au territoire national est, on l'a vu, un élément déterminant.

Le retournement de conjoncture lié à la crise qui a éclaté à la fin de l'année 2008 fait aujourd'hui planer beaucoup d'incertitudes sur le développement ultérieur des projets miniers au Sénégal Oriental. Après avoir vécu avec les «mirages » miniers, comment populations et décideurs locaux feront-ils face aux déceptions qui se profilent?

\section{BIBLIOGRAPHIE}

Collectif, 2000. Atlas du Sénégal, Paris, Les Editions Jeune Afrique aux Editions du Jaguar, 84 p.

D’Aquino P., 2002. « Le territoire entre espace et pouvoir : pour une planification territoriale ascendante ", in L'espace géographique 1-2002 (p. 3-22)

Debrie J., Steck B., 2001. «L'enclavement, réévaluation théorique et application à l'Afrique de l'Ouest », in L'Espace géographique 1-2001 (p. 26-36)

Diop M. C., 2002. La société sénégalaise entre le local et le global, Paris, Karthala, 723 p.

Gentil D., Husson B., 1996. « La décentralisation contre le développement local », in Observatoire permanent de la Coopération Française, Rapport 1996, Paris, Desclée de Brouwer (p. 59-77)

Greig I., 2006. Le Sénégal Oriental à l'aube du développement minier : quels enjeux pour les collectivités locales? Mémoire de master 1 de géographie dir. Magrin G., Ecole Normale Supérieure de Lettres et Sciences Humaines, $60 \mathrm{p}$.

Khennas S., 1993 Industrialisation, ressources minières et énergie en Afrique, Paris / Dakar, Karthala / CODESRIA, $348 \mathrm{p}$.

Magrin G., Ninot O., 2005. « Les zones soudaniennes du Tchad et du Sénégal : deux sud enclavés entre mondialisation et marginalisation », in Espace, populations, sociétés 2005-1 (p. 15-30)

Magrin G., 2006. « La décentralisation règlera les problèmes de l'Etat en Afrique ", in Courade G. (dir.) L'Afrique des idées reçues, Paris, Belin (p. 383-389)

Magrin G., Gautier D., 2006. Activités extractives et développement régional, rapport de mission à Tabakoto (ouest Mali), CIRAD, $19 \mathrm{p}$.

Ninot O., 2003. Vie de relations, organisation de l'espace et développement en Afrique de l'Ouest : la région de Tambacounda au Sénégal, thèse de doctorat en géographie dir. Lesourd M. et Lombard J., Université de Rouen, 460 p.

Observatoire de la décentralisation, 2003. Etat de la décentralisation en Afrique, Paris, Karthala-PDM, $357 \mathrm{p}$. 
Ouedraogo F., 1999. Etude sur le développement du secteur minier et l'environnement dans les pays de la ceinture sahélienne de l'Afrique de l'Ouest (Sénégal, Burkina, Mali et Niger), Dakar, Centre de Recherche pour le Développement International, $156 \mathrm{p}$.

Piveteau A., 2005. « Décentralisation et développement local au Sénégal. Chronique d'un couple hypothétique ", in Dubresson A., Faure P. A. (dir.) Décentralisation et développement local, un lien à repenser, Tiers-Monde $\mathrm{n}^{\circ}$ 181, tome XLXI, janvier-mars 2005.

Politique Africaine n 96 (décembre 2004) « Sénégal 2004, l'alternance et ses contradictions », Paris, Karthala, 230 p.

Totte M., Dahou T., Billaz R., 2003. La décentralisation en Afrique de l'Ouest, entre politique et développement, Paris, COTA-Karthala-ENDA GRAF, 400 p.

\section{NOTES}

1. La région doit constituer avec les communes et les communautés rurales une Agence Régionale de Développement. Son conseil d'administration est composé d'un représentant par collectivité locale. Le Président du Conseil Régional en est le président et nomme le directeur. Ses missions peuvent se résumer à quatre fonctions principales : appui technique aux collectivités locales, coordination et harmonisation des programmes de développement des collectivités locales, suivi - évaluation de la mise en œuvre des projets et programmes de développement des collectivités locales et maîtrise d'ouvrage déléguée. Les ressources proviennent théoriquement des contributions des collectivités locales, des subventions et du remboursement des frais relatifs aux prestations réalisées pour le compte des collectivités locales.

2. GIRARDEL : Groupe Interdisciplinaire de Recherche pour l'Appui à la planification Régionale et au Développement Local, université Gaston-Berger de Saint-Louis (www.ugb.sn/recherche/ girardel.htm ).

3. A titre de comparaison, un pays voisin grand producteur d'or comme le Mali produit plus de 60 tonnes d'or par an.

4. Pour donner un ordre de grandeur, les exportations mondiales de fer s'élevaient en 2005 à 720 millions de tonnes.

5. Il s'agit du gouvernement d'Abdoulaye Wade, leader du Parti Démocratique Sénégalais et principal opposant au régime de Senghor et de Diouf, élu Président en 2000. Le mouvement du « sopi » (changement) a fait naître beaucoup d'espoir.

6. Les enquêtes ont porté plus particulièrement sur trois communautés rurales du département de Kédougou : Khossanto, où sont implantées plusieurs sociétés d'exploration et d'exploitation industrielle et semi-industrielle de l'or, Saraya, où se trouvent plusieurs sociétés d'exploration de l'or et la plus grande partie du périmètre de la MIFERSO, enfin Bandafassi, où sont implantées les trois sociétés d'exploitation du marbre.

7. Les activités du port reposeraient principalement sur l'exportation des phosphates de Thiès et de Taiba ainsi que du fer du sud-est.

8. Important programme de lutte contre la pauvreté en milieu rural coordonné par le gouvernement du Sénégal et financé par la Banque Mondiale et le Fonds International de Développement Agricole. 


\section{RÉSUMÉS}

On assiste au Sénégal Oriental aux prémices d'un développement minier faisant intervenir des acteurs multiples et inégaux. Les difficultés rencontrées sur le terrain traduisent enjeux invisibles et tensions palpables. Il s'agit d'analyser la position fragile des collectivités locales dans un jeu d'acteurs complexe, placé sous le double signe de la mondialisation économique et d'une décentralisation inaboutie. Région et communautés rurales ont un rôle déterminant à jouer dans l'orientation des dynamiques territoriales, à l'articulation d'un horizon national trop lointain et d'un échelon ultra-local survalorisé.

Eastern Senegal is faced with the beginnings of a mining activities expansion that involves numerous and unequal players. The difficulties encountered during the survey reflect invisible stakes and palpable tensions. It's a matter of analyzing the fragile position of local authorities in a complex set of players, facing at the same time economic globalization and unfulfilled decentralization. The region and the rural communities have a determining part to play in the direction of the territorial dynamics, between a distant national level and a too emphasized ultra-local level.

\section{INDEX}

Keywords : Mines, enclosing, decentralization, local development, globalization, environment Mots-clés : Mines, enclavement, décentralisation, développement local, mondialisation, environnement

\section{AUTEUR}

\section{ISABELLE GREIG}

Isabelle Greig (greig.isabelle@gmail.com)est élève de l'Ecole Normale Supérieure de Lettres et Sciences Humaines, en Master 2 de géographie « Pays émergents et en développement » (Paris 1). 